\title{
MuSun Experiment: Measuring the Rate of Muon Capture on Deuteron
}

\section{Xiao Luo Boston University}

E-mail: xiaoluo@bu.edu

\begin{abstract}
The goal of the MuSun experiment at PSI is to measure the rate of muon capture on the deuteron with a precision of $1.5 \%$. This rate will be used to fix the low-energy constant that describes the two-nucleon weak axial current in Chiral perturbation theory. It will therefore calibrate evaluations of solar proton-proton fusion and neutrino-deuteron scattering(SNO experiment). MuSun forms part of the systematic program to achieve a new level of precision in confronting the theories of weak interactions, QCD and few body physics. MuSun inherits some of the well developed techniques and apparatus from a successful measurement of the rate for muon capture on the proton, the MuCap experiment, also performed at PSI. As in MuCap, MuSun uses a TPC as an active target. To optimize the molecular kinetics, its ultra-pure deuterium gas is kept at $31 \mathrm{~K}$. The status of the hardware and details of the data analysis for a high statistics run taken in 2013 will be presented.
\end{abstract}

16th International Workshop on Neutrino Factories and Future Neutrino Beam Facilities - NUFACT2014, 25 -30 August, 2014

University of Glasgow, United Kingdom 


\section{Introduction}

The goal of MuSun experiment is to measures the rate $\Lambda_{d}$ for the semileptonic weak process $\mu^{-}+d \rightarrow v_{\mu}+n+n$ (1) with better than $1.5 \%$ precision. $\Lambda_{d}$ is the capture rate from the doublet hyperfine state of the muonic deuterium atom in its $1 \mathrm{~S}$ ground state. The strong motivations for this measurement include

- $\mu d$ capture is the simplest process on a nucleus which both can be calculated and measured to a high degree of precision.

- Past measurement of the $\mu d$ capture rate are consistent with each other, but not very precise, with errors in $6-10 \%$ range [1]. Those experimental measurements are not accurate enough to explain the discrepancy of different theoretical calculations.

- The $\mu d$ capture rate could be directly translated to the N3LO Low energy constant(LEC) $\hat{d}_{R}$ in Chiral Perturbation Theory(ChPT).

- Several other important interactions, such as pp fusion in the sun, $v d$ scattering, nn scattering, etc will benefit from improving the experimental precision of $\hat{d}_{R}$.

\section{Theory}

The most natural theoretical framework for MuSun is ChPT, which is the effective field theory of the QCD in the low energy region, where $Q / \Lambda_{\chi} \ll 1$. $Q$ is the energy of the reaction and $\Lambda_{\chi} \simeq 4 \pi f_{\pi} \simeq m_{N}$ with $m_{N}$ is the nucleon mass [2]. As mentioned previously in sect. 1, ChPT is a model independent framework and cross sections of many low energy processes could be calculated to high precision with the fixed low energy constants (LEC). So far, up to the N3LO, there is only one unknown LEC, $\hat{d}_{R}$. It appears in the $2 \mathrm{~N}$ axial current and is best determined from the experiment. Before, $\hat{d}_{R}$ was theoretically fixed by fitting the triton bending energy and lifetime. But this method involves two free parameters which are correlated with each other, thus the result has large error bar. Nowadays, to fix this constant more accurately, the cleaner and simpler process $\mu d$ capture is chosen. The most recent $\mu d$ capture rate in the doublet state, calculated by L.E. Marcucci and collaborators, is found to be 399(3) s $\mathrm{s}^{-1}$ and 400(3) $\mathrm{s}^{-1}$ with different neutron-neutron scattering length.[1]

\section{Experimental design}

\subsection{Lifetime technique}

The products of the $\mu d$ capture reaction are all neutral particles, which are difficult to detect. Instead we use the so-called lifetime technique, where we infer the capture rate from comparing the measured lifetime of $\mu^{+}$and $\mu^{-}$in our deuterium target. $\mu^{+}$disappears only through muon decay, and $\mu^{-}$disappears through both decay and capture. Therefore $\mu d$ capture rate will be the difference between positive and negative muon disappearance rates. ${ }^{1}$ The observables of our

\footnotetext{
${ }^{1}$ We will use the world average muon decay rate as the $m u^{+}$disappearance rate
} 
experiment are decay electron time and muon entrance time. We fill our lifetime histograms with the time difference between the two. The Muon disappearance rate is simply the decay constant in a fit to an exponential curve.

In order to achieve the $1.5 \%$ precision, the collection of $10^{10}$ "good" muon events is required. Up to now, we finished the data taking from three major production runs took place in 2011, 2013 and 2014 and collect a few billion events each run during 10 weeks of run period. We are planing to have our final run in 2015 to well exceed statistical requirement.

\subsection{Detector System}

The MuSun experiment takes advantage of the intense low-energy muon beam in the $\pi E 1$ area of the Paul Sherrer Institut (PSI) in Switzerland. At the optimal deuterium density and temperature, stopping the muons near the center of the TPC requires a beam momentum of approximately $40 \mathrm{MeV} / \mathrm{c}$. We tune the beam line magnets to focus the muon beam at the muon entrance detector with the rate about $27 \mathrm{kHz}$; the corresponding stopping rate in the deuterium target is about $12 \mathrm{kHz}$. The MuSun beam line also includes electronic separator and kicker. The separator is a velocity selection device which helps to separate the beam electrons from muons. The separator is also used to move the beam in the vertical direction. When energized, the high-voltage kicker prevents muons from entering the TPC. To maximize our usable rate, we run in "muon on request" mode. Whenever a muon enters the detector system, the signal from an entrance scintillator is used to trigger the kicker. Its deflecting voltage prevents the entrance of subsequent muons from the continuous cyclotron beam.

The MuSun detector system includes three major components, see figure 1: the muon entrance counters ( $\mu$ Entrance), the time projection chamber (TPC), and electron detectors(Edet).

- $\mu$ Entrance: consists MuSC, a thin scintillator to define the muon time; MuSCA, a scintillator with a $3.5 \mathrm{~cm}$ diameter whole in the center, to define the beam size and veto the off-centered events; and MuPC, a $5 \mathrm{~cm} \times 5 \mathrm{~cm}$ wire chamber to locate the XY position of the muons.

- TPC: A ionization chamber filled with ultra-pure deuterium gas.The gas density is approximately $6 \%$ of that for liquid hydrogen. In order to lower the impurity concentration and optimize the $\mu d$ doublet state population, our TPC runs at a temperature of $31 \mathrm{~K}$. $80 \mathrm{kV}$ high voltage is applied between cathode and anode, so each negative muon will ionize the deuterium gas along its track. The ionization electrons will descend to the anode pad plane with drift velocity of approximately $0.5 \mathrm{~cm} / \mu \mathrm{s}$. The pad plane is segmented into 6 (transverse) $\times$ 8 (longitudinal $)=48$ small pads, each of dimension $16 \mathrm{~mm} \times 18 \mathrm{~mm}$. We use the TPC to reconstruct the muon track in 3 dimensions. For our final analysis, we choose only "good stops", where the muon stops well away from the chamber walls. With the newly-developed pre-amplifier, we have also achieved the energy resolution of $10 \mathrm{keV}[3]$. Better separation between the noise and the signal makes it easier to define the muon tracks and also helps with particle-identification. 


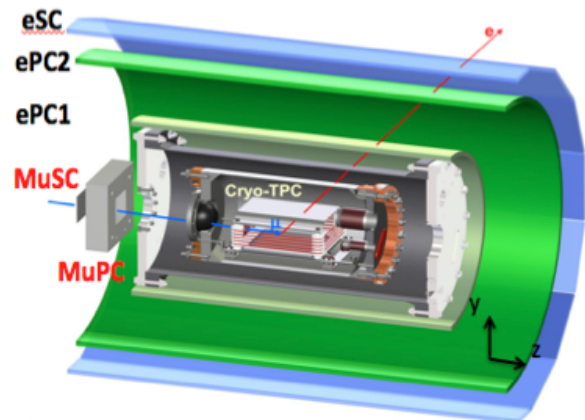

(a)

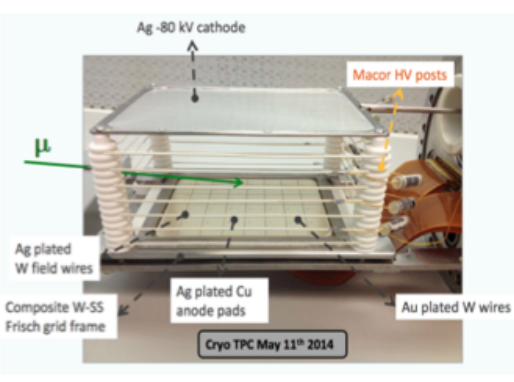

(b)

Figure 1: (a) is the drawing of the MuSun whole detector system, we get the $t_{\mu}$ from the MuSC, and $t_{e}$ from the eSC. (b) is the picture of the new TPC for 2014 run

- Edet: A $3 \pi$ detector, including two layers of wire chamber (ePC1 and ePC2) and the 16 two-layer scintillator strips of the eSC. The former is used to reconstruct the decay-electron track of the Michel electron and the latter, to determine its decay time.

\subsection{Data analysis}

The MuSun collaboration has developed a two-tier analysis package consisting of two programs, MU and MTA.

- MU: The raw data in the MIDAS format is first processed by MU. Due to the detector responding time difference and various cable lengths, we use time autocorrelation data to align the detector signals in time. To align the three electron detectors in space, we use through-going cosmic rays.

Another task, also the main goal of the MU, is to form physics objects from each detector and prepare the "MuSun" event. The MuSun event is seeded by the $\mu$ Entrance event and includes all detector information in a time window of $[-30,+30] \mu s$ around the entrance event.

- MTA: "MuSun" events are written by MU and analyzed in MTA. Good events, destined for the lifetime histograms, are chosen on the basis of timing and fiducial volume cuts. The most difficult task is to choose events where the muon track stops in the deuterium gas. Most of the time, we get a clean TPC event, such as that shown in figure 2. The track consists of a straight line of pads and a clear muon stop inside of the TPC's fiducial volume. We also observe the Bragg-curve in the succession of the pulse waveform in the time display. However, due to scattering and the beam's spacial and momentum distribution, muons sometimes stop in peripheral regions where they might capture on low- $\mathrm{Z}$ and medium- $\mathrm{Z}$ materials. Out fiducial volume cut removes the stops in such problematic regions. The fiducial volume is defined as inner region one pad away from the border of the pad plane in $\mathrm{X}$ and $\mathrm{Z}$, and central $4 \mathrm{~cm}$ vertically midway between cathode and anode. Additionally, to exclude random noise triggers, we require the muon track length be at least 3 pads long in Z. Several clustering algorithms are currently being tested. 

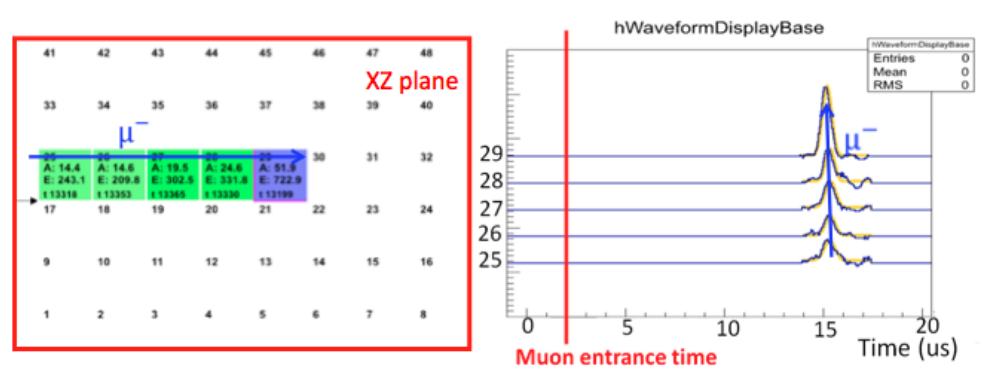

Figure 2: A typical TPC event, left is the pad plane display showing the $\mathrm{XZ}$ coordinates of the muon track and color coded energy information; The right is the waveform display, providing the height of the muon track and waveform for particle type identification.

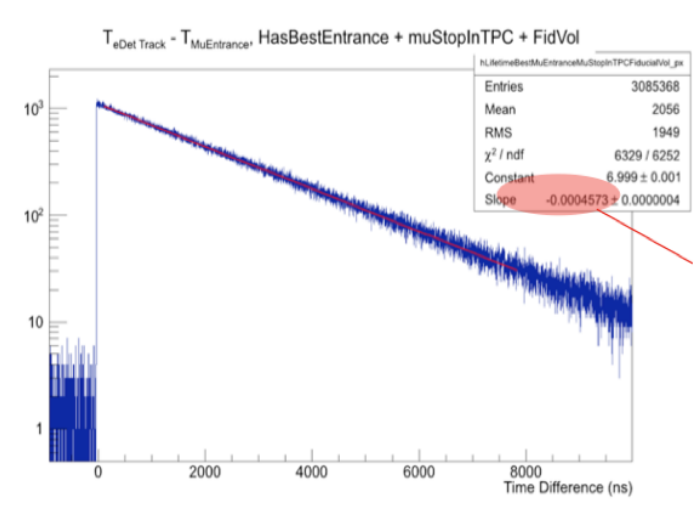

Figure 3: lifetime histogram, the muon disappearance rate $\Lambda_{\mu}$ is extracted by fitting a 3 parameter exponential function

Muon-electron pairs are formed from tracks that survive the cuts in MTA. We then fill the lifetime histogram with the time difference between electron and muon. See figure 3 The time spectrum should be well-described by an exponential decay sitting on a flat background, and the decay constant is the muon disappearance rate. To prevent bias we blind ourselves to the true frequency of the two experimental clocks - during the run and during the subsequent analysis. The frequency of the experimental clocks will only be revealed when the analysis is complete and will only be used to determine the final result.

- Monte-Carlo: One big advantage of the MuSun analysis, in comparison to the MuCap analysis, is a complete Monte Carlo simulation package. We use GEANT4 to generate the muon beam and include all the experimental detectors as sensitive detectors. The ideal simulation data is then processed through a separate detector response package, designed to include realistic noise and detector inefficiency. Because of its importance in assessing systematic errors, much time was spent on tuning the simulation model to better match the experimental data, particularly the parameters of the beam distribution and the details of the TPC response. The MuSun Monte Carlo is now a powerful tool for determining deficiencies in the analysis and, where data-driven approaches are impossible for estimating the scale of various systematic errors. 


\section{Systematics}

The high precision measurement of the $\mu d$ capture rate in MuSun requires that the overall systematic error be less than $5 \mathrm{~Hz}$. Our Systematic effects could be grouped into two types: physics processes, meaing other than muon decay and $\mu d$ capture, the sources that contribute to the muon disappearance rate; and artificial systematics errors created by a time-dependence in the acceptance of detector system and analysis selection after cuts.

To handle errors of the first kind, we have various options. If the extra contribution to the muon disappearance rate is small in amplitude but very high in rate, we can simply delay the start of our fit until that contribution is negligible. If the extra contribution arises from a well-understood source, we can introduce another exponential decay term, with fixed rate and unknown amplitude, into our fit function. If the time dependence of the extra source is unknown, its decay rate can be included as a fit parameter. However, the introduction of extra parameters weakens the statistical power of the data and unless they describe the data well, the new parameters can distort our results for the capture rate.

As for the second type, any time-dependence in the detector acceptance, usually created as the result of experimental cuts, results in a direct systematic error. Time-dependent acceptance is addressed using both data-driven and simulation techniques. We look for time sensitivity in the cuts by seeing how the fitted disappearance rate $\Lambda_{\mu}$ varies with cut value that we choose. If the fitted rate $\Lambda_{\mu}$ does not change as we vary the cut over some range of values, then we choose the value which maximizes the statistical power of our data. If $\Lambda_{\mu}$ does change, we can sometimes extrapolate the trend to get the correction from the true value $\Delta \Lambda_{\mu}$ with the given cut value. In other cases, purely data-driven approaches are inadequate by themselves. In this case, we rely on simulation to predict the scale of the systematic correction, and to provide guidance for improving our reconstruction algorithm. Our final systematic error estimates often require a combination of both. Two examples are described in more detail below: systematic errors from chemical impurities provide an example of the first kind, and fusion interference, one of the most complicated issues for the MuSun analysis, is a good example of the second.

\subsection{Impurity}

The cryogenic Circulating Hydrogen Ultra-High Purification System (CHUPS) continuously removes impurities from the deuterium gas in the TPC. The equilibrium vapor pressure at low TPC temperature sets a very low upper limit on impurity concentrations. However, even though the impurities are largely suppressed, they still provide an extra muon disappearance channel and can therefore modify the measured capture rate. The error bar of this modification is defined by the inaccuracy of impurity concentrations.

For MuSun, running at $31 \mathrm{~K}$, the main impurities are $\mathrm{N}_{2}$. We monitor its concentration using two methods: gas chromatography and direct detection. Both methods are currently under calibration and the goal is to reach less than $0.5 \mathrm{ppb}$ precision. To give a rough idea, $1 \mathrm{ppb} \mathrm{N}_{2}$ will lead to a $2 \mathrm{~Hz}$ correction in disappearance rate. The direct detection method take advantage of mono-energetic ${ }^{3} \mathrm{He}$ signal (generated from the muon catalyzed fusion, see sect. 4.2) to optimize energy resolution, as shown in figure 4 . With optimal cuts, the carbon recoil signal produced by muon capture on the nitrogen will appear as a bump in ${ }^{3} \mathrm{He}$ spectrum. The simulation was used to 


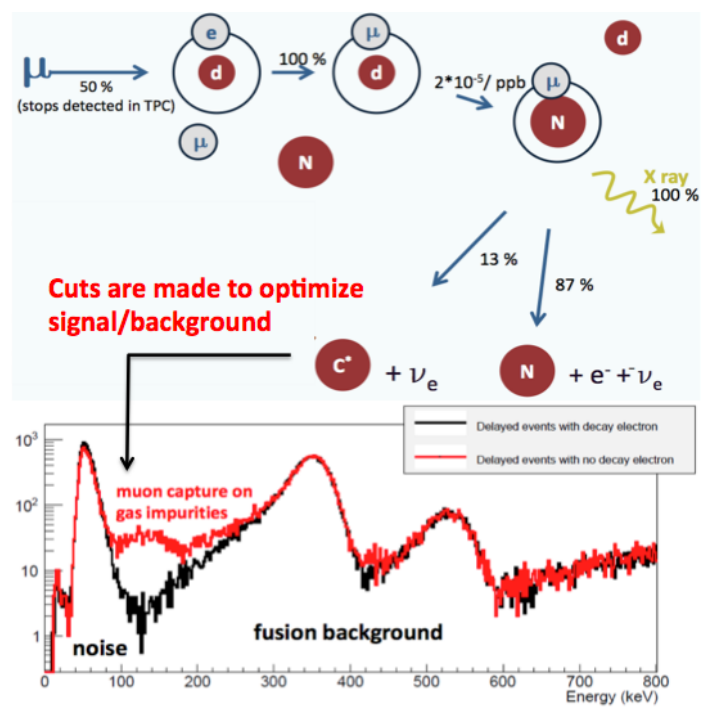

Figure 4: direct detection of muon capture on impurities such as $\mathrm{N}_{2}$ and $\mathrm{O}_{2}$

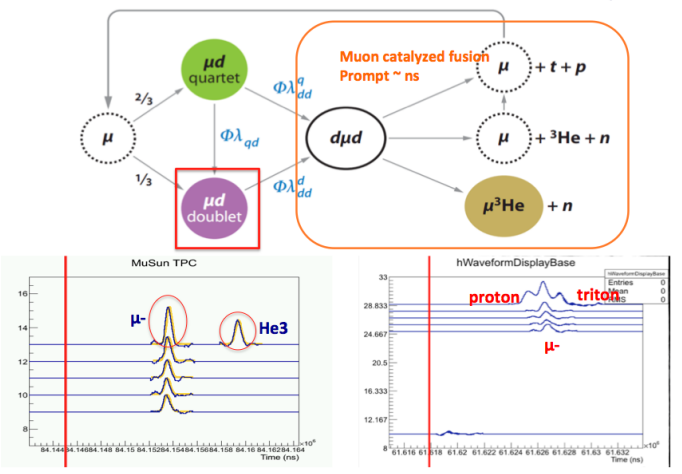

Figure 5: The upper diagram is the kinetics of the processes after muon stops. And the lower are the event displays of muon catalyzed fusion: $\mu^{-}+d \rightarrow^{3} H e 3+n+\mu^{-}$(left), $\mu^{-}+d \rightarrow p+t+\mu^{-}$(right).

distinguish the number of actual $\mathrm{N}_{2}$ capture events from the background events such as neutrondeuteron scattering. Preliminary result indicates that the contribution of this background channel to the "impurity bump" is compatible with that from $1 \mathrm{ppb} \mathrm{N}_{2}$ concentration.

\subsection{Fusion interference}

Figure 5 is a kinetics diagram showing the major processes that a muon could experience after stopping in the TPC. About $10 \%$ of all the valid muon stops will experience muon catalyzed fusion. The fusion products generate extra pulses in the stop pad or its surrounding pads, which affects our decision of a "good muon stop".

We apply the fiducial volume (FV) cut to veto events that stop the outmost rows of pads. However, fusion signals could push a muon stop from outside to inside of the FV, or vice versa. Unfortunately, this migration caused by the fusion is also time dependent. To check the scale of this event migration, we generated $2 \times 10^{7} \mu^{-}$events and processed them through the detector response with and without fusion products. Then, after analyzing the simulated data with the experiment's 
tracking algorithm and subjecting the results to our FV cut, we compared results of lifetime fits. Overall, we found for about $500 \mathrm{ppm}$ of all muon stops, fusion interference tends to push the "bad" event into the FV (900ppm outflow and 1400ppm inflow). This net migration causes a approximately $50 \mathrm{~Hz}$ decrease of the disappearance rate. Because our systematic error budget is only $5 \mathrm{~Hz}$, the effect of fusion interference is clearly of great concern. We are currently developing a combined data-driven and simulation approach to reduce this systematic correction as much as possible.

\subsection{Other Systematics}

There are many more other systematic corrections we need to make to $\Lambda_{\mu}$. This includes the correction of the capture from $\mu d$ quartet state. The populations of $\mu d_{d o u b l e t}$ and $\mu d_{\text {quartet }}$ states will be defined from the neutron detector analysis. Pileup, when two muons overlapping the detector system is also problematic. Because pileup is most likely in the first 600ns after the muon stop, before the kicker is energized, it can cause a distortion of the measured disappearance rate. Although our entrance detectors are very sensitive to pileup, even small levels of unseen pileup can create significant systematic errors. We still need to quantify the level of pileup and its effect on our measurement of the decay constant. Electron interference, where ionization from a decay electron changes our interpretation of data from a muon stop, creates systematic errors that are similar in character to those produced by fusion interference. Simulation analysis shows that the effect is smaller but not negligible.

\section{Summary}

MuSun is measuring the rate of muon capture on deuteron in doublet state. We have finished taking three big production runs at PSI with each run period about 3 months. We are planning to stage our final production run in 2015 . The collaboration is currently working hard on the data analysis. We are estimating systematic errors, using both data-driven and simulation techniques. We expect to announce our first result, from data taken in 2011, next year. It will have relatively large systematic errors. Results from the 2013 and 2014 datasets are expected in two years.

\section{Acknowledgment}

I gratefully acknowledge the work of all of our MuSun collaborators, and I thank them to give me this opportunity to participate this conference and allow me to present their work. Special thanks to my advisor Prof. Robert Carey for editing and proofreading this proceeding.

\section{References}

[1] Laura Elisa Marcucci, Muon Capture On the Deuteron and ${ }^{3} \mathrm{He}$ : A Proposal Review arXiv:1112.0113v1, page 4 (2011)

[2] MuSun collaboration, V.Andreev et al., Muon capture on the deuteron - the MuSun experiment arXiv:1004.1754, page 7 (2008)

[3] R.A.Ryan et al, Design and operation of a cryogenic charge-integrating preamplifier for the MuSun experiment, arXiv:1404.3251v2 (2014) 